Case Report

\title{
Nodular Lymphocyte-Predominant Hodgkin Lymphoma in Progressive Transformation of Germinal Centers
}

\author{
C. Eric Bailey, ${ }^{1}$ Francesca Jung, ${ }^{2}$ Benjamin Addicks, ${ }^{1}$ Olukemi A. Esan, ${ }^{3}$ and \\ Brian Kellermeyer ${ }^{1}$ \\ ${ }^{1}$ Department of Otolaryngology, West Virginia University Health Science Center, Morgantown, WV, USA \\ ${ }^{2}$ School of Medicine, West Virginia University, Morgantown, WV, USA \\ ${ }^{3}$ Department of Pathology, West Virginia University Health Science Center, Morgantown, WV, USA
}

Correspondence should be addressed to Brian Kellermeyer; bmkellermeyer@hsc.wvu.edu

Received 26 July 2017; Accepted 8 October 2017; Published 14 November 2017

Academic Editor: Abrão Rapoport

Copyright ( 2017 C. Eric Bailey et al. This is an open access article distributed under the Creative Commons Attribution License, which permits unrestricted use, distribution, and reproduction in any medium, provided the original work is properly cited.

Nodular lymphocyte-predominant Hodgkin lymphoma is an uncommon variant of Hodgkin lymphoma. Progressive transformation of germinal centers has been associated with and can develop prior to, concurrent with, or after the diagnosis of nodular lymphocyte-predominant Hodgkin lymphoma. We present a patient with a history of progressive transformation of germinal centers of the right parotid who presented 4 years later with ipsilateral parotid mass and cervical adenopathy. Knowledge of her previous diagnosis raised our concern for lymphoma, influenced our surgical management, and spared the patient additional surgery with risk of facial nerve injury inherent in revision parotidectomy.

\section{Introduction}

Masses of the parotid gland warrant thorough investigation, as $15-32 \%$ of parotid gland masses are found to be malignant [1]. The course of malignant parotid gland tumors or other salivary gland tumors is highly variable, but they have the potential to be aggressive and result in high levels of morbidity and mortality. Painless swellings of the parotid gland, especially those involving the facial nerve, necessitate pathologic examination with either fine-needle aspiration (FNA) or ultrasound-guided core needle biopsy. FNA, in particular, has been found to be highly specific in determining benign versus malignant parotid gland lesions [2] and is strongly recommended when the mass could possibly represent mimickers such as TB, lymphoma, or enlarged lymph nodes [1].

Alternatively, progressive transformation of germinal centers (PTGC) typically presents as an asymptomatic enlarged lymph node of the head and neck [2]. First reported by Lennert and Müller-Hermelink, PTGC is a benign condition that can be mistaken for malignant processes such as nodular lymphocyte-predominant Hodgkin lymphoma
(NLPHL) and follicular lymphoma [3]. The name itself somewhat describes the entity; the germinal center expands as mantle zone lymphocytes infiltrate the germinal center and blur the margin between the mantle zone and germinal center $[2,4]$. Although the pathogenesis of PTGC is unclear and likely multifactorial, it is believed to be a state of follicular hyperplasia following reactive stimuli $[5,6]$. PTGC and nodular lymphocyte-predominant Hodgkin lymphoma (NLPHL) are often found concurrently, or with one entity preceding the other. While PTGC may precede NLPHL, some studies have shown that patients with PTGC did not show an increased risk for developing NLPHL, making it uncertain whether the clonal population of cells comprising NLPHL arises from PTGC directly $[2,6]$.

PTGC presents most often in males with a $3: 1$ male to female ratio and generally presents as painless, persistent lymphadenopathy [7]. Cervical chain lymph nodes are most frequently affected by PTGC, with nearly $50 \%$ of cases, followed by inguinal (25\%) and axillary (22\%) [1]. Approximately $3.5-10 \%$ of patients presenting with painless, persistent lymphadenopathy are diagnosed with 


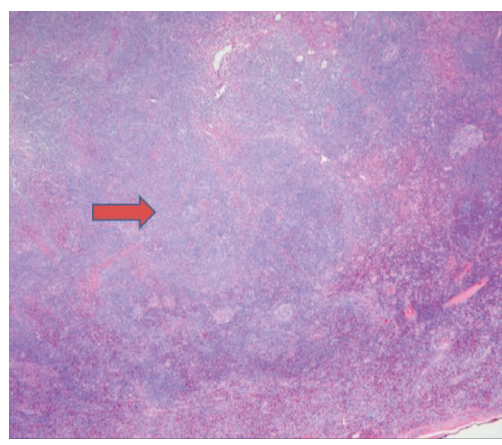

(a)

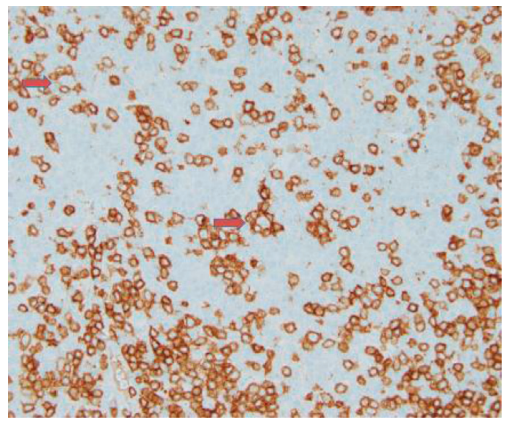

(d)

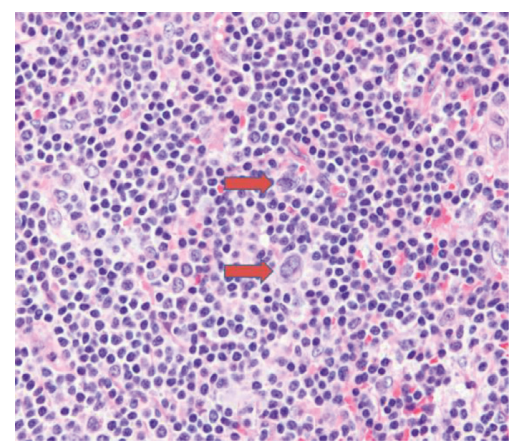

(b)

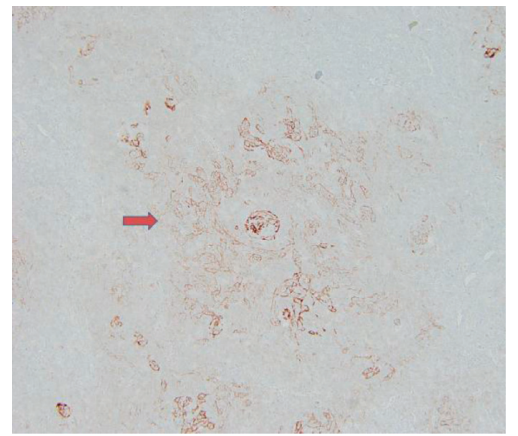

(e)

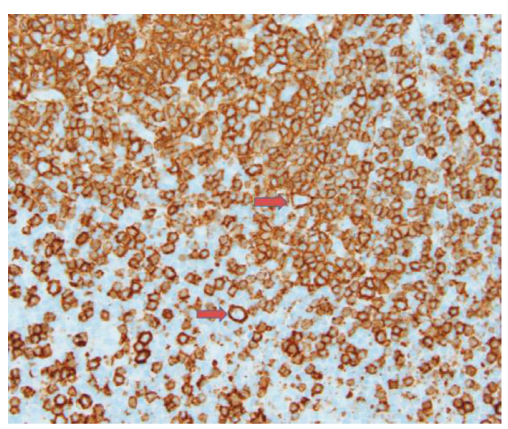

(c)

FIgURE 1: (a) Low-power H\&E staining showing prominent nodules in the background of reactive lymphoid tissue. (b) Interspersed large "popcorn” lymphocyte-predominant cells. (c) Large popcorn cells are positive for CD20. (d) CD3-positive T cells form rosettes around the large popcorn cells. (e) CD21 staining showing expanded follicular dendritic network in the large nodule.

PTGC [8]. In prior reports, anywhere between 16 and $35 \%$ of PTGC are associated with NLPHL $[7,9,10]$.

We present a case of a 39 -year-old female patient in which the knowledge of prior diagnosis of PTGC influenced workup and management when she presented with recurrent parotid mass and ipsilateral lymphadenopathy.

\section{Case Report}

A 39-year-old female presented to the clinic with right neck lymphadenopathy and an apparent ipsilateral parotid mass. Her previous history was significant for ipsilateral superficial parotidectomy and neck dissection performed at another institution. Per the patient, the pathology report from her parotidectomy showed no lymphoma or other malignancy, but she did mention that she was sent to an oncologist after surgery. After a four-year symptom-free interval, she presented to us with recurrent right parotid mass, neck lymphadenopathy, fatigue, and weight loss. Facial nerve was intact bilaterally.

Given the recurrent nature, presence of lymphadenopathy, and the patient's age, suspicion of malignancy was high. Multiple fine-needle aspiration biopsies of the cervical lymph nodes and parotid mass failed to establish diagnosis. When pathology was obtained from her previous surgery, the report described PTGC. After review of the pathology from the previous procedure, the decision was made to proceed with excisional biopsy of level II cervical lymph node, sparing the previously operated parotid space with inherent risk of facial nerve injury in revision parotidectomy. Pathology revealed NLPHL in a portion of the node, adjacent to areas of PTGC.

The patient had an uncomplicated postoperative recovery without facial nerve injury or other morbidities. Postoperative PET/CT showed hypermetabolic lymphadenopathy of the right neck, parotid, and right inguinal areas. She was treated with chemotherapy with good response to treatment.

\section{Pathology Discussion}

It is well known that progressive transformation of germinal centers can mimic nodular lymphocyte-predominant Hodgkin lymphoma both clinically and histologically [3]. While PTGC may be a precursor of NLPHL, many patients with PTGC do not develop NLPHL later in life [11]. Of note, PTGC may be present in the same lymph node as NLPHL; thus, careful sampling is required to exclude malignancy in lymph nodes involved by PTGC [12]. Progressively transformed follicles are similar to NLPHL nodules in that they are composed of nonneoplastic small mantle B cells. However, interspersed hyperplastic follicles are typically present, which is not usual in NLPHL [3]. In addition, the nodules do not contain the neoplastic lymphocyte-predominant (LP) cells seen in NLPHL. These LP cells are larger atypical lymphocytes with lobulated nuclei resembling kernels of popped corn also referred to as popcorn cells [13]. By immunohistochemical staining, the LP cells express CD20 and CD45 while being negative for CD15 
TABLE 1: Summary of literature.

\begin{tabular}{|c|c|c|c|c|c|c|}
\hline Study & $\begin{array}{c}\text { Year of } \\
\text { publication }\end{array}$ & $\begin{array}{c}\text { Cases of } \\
\text { PTGC }\end{array}$ & $\begin{array}{l}\text { NLPHL diagnosis, } \\
\text { total }\end{array}$ & $\begin{array}{l}\text { PTGC before } \\
\text { NLPHL }\end{array}$ & Concurrent & $\begin{array}{l}\text { NLPHL before } \\
\text { PTGC }\end{array}$ \\
\hline Poppema et al. [15] & 1979 & 5 & 5 & 2 & 1 & 2 \\
\hline Osborne and Butler [6] & 1984 & 50 & 12 & 0 & 5 & 7 \\
\hline Burns et al. [12] & 1984 & 36 & 36 & 2 & 31 & 3 \\
\hline Crossley et al. [16] & 1987 & 1 & 1 & 1 & 0 & 0 \\
\hline Hansmann et al. [11, 17] & 1990 & 66 & 11 & 3 & 4 & 4 \\
\hline Ferry et al. [18] & 1992 & 5 & 0 & 0 & 0 & 0 \\
\hline Verma et al. [19] & 2002 & 2 & 0 & 0 & 0 & 0 \\
\hline Kojima et al. [2] & 2003 & 42 & 0 & 0 & 0 & 0 \\
\hline Licup et al. [20] & 2006 & 5 & 0 & 0 & 0 & 0 \\
\hline Shaikh et al. [14] & 2012 & 29 & 1 & 1 & 0 & 0 \\
\hline Chang et al. [9] & 2015 & 1 & 0 & 0 & 0 & 0 \\
\hline Özkan et al. [10] & 2016 & 33 & 3 & 0 & 0 & 1 \\
\hline Total & & 275 & 69 & 9 & 43 & 17 \\
\hline
\end{tabular}

and CD30. In addition, PD-1-positive T cells form rosettes around LP cells in NLPHL [13].

\section{Discussion}

A parotid mass with lymphadenopathy in an adult patient necessitates evaluation. While FNA is commonly employed in the workup of salivary lesions or lymphadenopathy, it is often nondiagnostic when dealing with hematogenous malignancies, with core biopsy or excisional biopsy often needed to provide adequate sample for analysis of node architecture and diagnosis. This particular patient presented with worrisome progressive enlargement, weight loss, and concern for malignancy. At the initial clinic encounter when pathology from the prior surgery was unavailable, the lymphadenopathy prompted concern for carcinoma ex pleomorphic (given her previous parotidectomy), metastatic squamous cell carcinoma, mucoepidermoid carcinoma, or some other primary salivary malignancies. After previous pathology was obtained, the noted association between PTGC and NLPHL was considered when FNA as a diagnostic modality failed. This influenced surgical decision-making and led us to undertake excisional biopsy of a cervical node rather than parotidectomy. Based on frozen section which suggested hematologic malignancy was most likely, this spared the patient an extensive revision parotidectomy and the associated morbidity of such a surgery.

In a thorough English language literature search, the percentage of cases of progressive transformation of germinal centers associated with nodular lymphocyte-predominant Hodgkin lymphoma was $25.1 \%$, as shown in Figure 1 . The diagnosis of NLPHL could precede, antecede, or appear concurrently with a diagnosis of PTGC. Prior reports found the association to be between 16 and 35\% [7, 11]. A PTGC diagnosis prior to a diagnosis of NLPHL, as in the case of our patient, is rare. In our literature review, a total of 9 reported cases out of 275 (3.27\%) were found to have PTGC preceding diagnosis of NLPHL. However, the association overall is sufficient to consider following patients with either PTGC or NLPHL for development of the alternative disease. Additionally, as the rate of concurrent PTGC and NLPHL has the strongest association, it is important to be certain of a single diagnosis when either is discovered. A diagnosis of NLPHL succeeding PTGC may appear anywhere from less than one year to 13 years after the diagnosis of PTGC, and the fear of diagnosis may lead to multiple repeat biopsies [14]. Although our patient's NLPHL was found in the same location as her prior PTGC, NLPHL may be found in separate lymph nodes, most commonly of the cervical chain [7]. There is no consensus at this point regarding the appropriate follow-up interval or imaging for PTGC. Further study should be considered to evaluate the proper interval and duration of follow-up for patients with PTGC (Table 1).

\section{Disclosure}

The manuscript was presented as an abstract at Combined Otolaryngology Spring Meetings 2016.

\section{Conflicts of Interest}

The authors declare that they have no conflicts of interest.

\section{References}

[1] M. Guzzo, L. D. Locati, F. J. Prott, G. Gatta, M. McGurk, and L. Licitra, "Major and minor salivary gland tumors," Critical Reviews in Oncology/Hematology, vol. 74, no. 2, pp. 134-148, 2010.

[2] M. Kojima, S. Nakamura, T. Motoori et al., "Progressive transformation of germinal centers: a clinicopathological study of 42 Japanese patients," International Journal of Surgical Pathology, vol. 11, no. 2, pp. 101-107, 2003.

[3] L. B. Smith, "Nodular lymphocyte predominant Hodgkin lymphoma diagnostic pearls and pitfalls," Archives of Pathology \& Laboratory Medicine, vol. 134, no. 10, pp. 1434-1439, 2010. 
[4] C. C. Chang, V. Osipov, S. Wheaton, S. Tripp, and S. L. Perkins, "Follicular hyperplasia, follicular lysis, and progressive transformation of germinal centers: a sequential spectrum of morphologic evolution in lymphoid hyperplasia," American Journal of Clinical Pathology, vol. 120, no. 3, pp. 322-326, 2003.

[5] C. C. Liu, A. R. Jethwa, S. S. Khariwala, J. Johnson, and J. J. Shin, "Sensitivity, specificity, and posttest probability of parotid fine-needle aspiration," Otolaryngology-Head and Neck Surgery, vol. 154, no. 1, pp. 9-23, 2016.

[6] B. M. Osborne and J. J. Butler, "Clinical implications of progressive transformation of germinal centers," American Journal of Surgical Pathology, vol. 8, no. 10, pp. 725-733, 1984.

[7] J. Hicks and C. Flaitz, "Progressive transformation of germinal centers: review of histopathologic and clinical features," International Journal of Pediatric Otorhinolaryngology, vol. 65, no. 3, pp. 195-202, 2002.

[8] M. W. Miller, K. M. Gatter, S. B. Cannady, and M. K. Wax, "Progressive transformation of germinal centers (PTGC) in the head and neck," Laryngoscope, vol. 120, no. 4, p. S168, 2010.

[9] C. A. Chang, B. Kumar, and D. Nandurkar, "A case report of high 18F-FDG PET/CT uptake in progressive transformation of the germinal centers," Medicine, vol. 94, no. 23, p. e412, 2015.

[10] M. C. Özkan, N. Özsan, M. Hekimgil, G. Saydam, and M. Töbü, "Progressive transformation of germinal centers: single-center experience of 33 Turkish patients," Clinical Lymphoma Myeloma and Leukemia, vol. 16, pp. S149-S151, 2016.

[11] M. L. Hansmann, C. Fellbaum, P. K. Hui, and P. Moubayed, "Progressive transformation of germinal centers with and without association to Hodgkin's disease," American Journal of Clinical Pathology, vol. 93, no. 2, pp. 219-226, 1990.

[12] B. F. Burns, T. V. Colby, and R. F. Dorfman, "Differential diagnostic features of nodular L\&H Hodgkin's disease, including progressive transformation of germinal centers," American Journal of Surgical Pathology, vol. 8, no. 4, pp. 253-262, 1984.

[13] S. H. Swerdlow, E. Campo, N. L. Harris et al., "Tumours of haematopoietic and lymphoid tissues," in World Health Organization Classification of Tumours, France IARC Press, Lyon, France, pp. 323-325, 2008.

[14] F. Shaikh, B. Y. Ngan, S. Alexander, and R. Grant, "Progressive transformation of germinal centers in children and adolescents: an intriguing cause of lymphadenopathy," Pediatric Blood \& Cancer, vol. 60, no. 1, pp. 26-30, 2012.

[15] S. Poppema, E. Kaiserling, and K. Lennert, "Hodgkin's disease with lymphocytic predominance, nodular type (nodular paragranuloma) and progressively transformed germinal centres: a cytohistological study," Histopathology, vol. 3, no. 4, pp. 295-308, 1979.

[16] B. Crossley, A. Heryet, and K. C. Gatter, "Does nodular lymphocyte predominant Hodgkin's disease arise from progressively transformed germinal centres? A case report with an unusually prolonged history," Histopathology, vol. 11, no. 6, pp. 621-630, 1987.

[17] M. L. Hansmann, E. Gödde-Salz, P. K. Hui, H. K. MüllerHermelink, and K. Lennert, "Cytogenetic findings in nodular paragranuloma (Hodgkin's disease with lymphocytic predominance; nodular) and in progressively transformed germinal centers," Cancer Genetics and Cytogenetics, vol. 21, no. 4, pp. 319-325, 1986.

[18] J. A. Ferry, L. R. Zukerberg, and N. L. Harris, "Florid progressive transformation of germinal centers. A syndrome affecting young men, without early progression to nodular lymphocyte predominance Hodgkin's disease," American Journal of Surgical Pathology, vol. 16, no. 3, pp. 252-258, 1992.

[19] A. Verma, W. Stock, S. Norohna, R. Shah, B. Bradlow, and L. C. Platanias, "Progressive transformation of germinal centers," Acta Haematologica, vol. 108, no. 1, pp. 33-38, 2002.

[20] A. T. Licup, P. Campisi, B. Y. Ngan, and V. Forte, "Progressive transformation of germinal centers: an uncommon cause of pediatric cervical lymphadenopathy," Archives of Otolaryngology Head \& Neck Surgery, vol. 132, no. 7, pp. 797-801, 2006. 


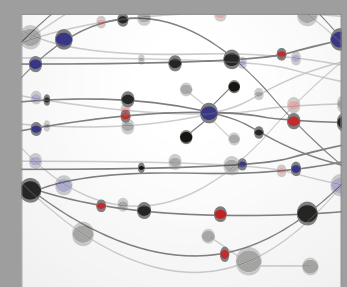

The Scientific World Journal
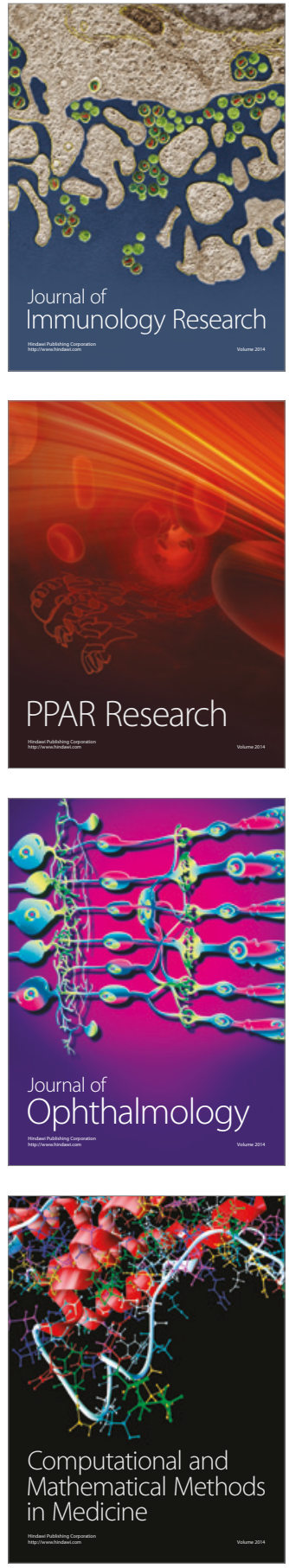

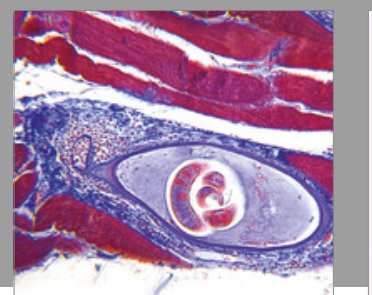

Gastroenterology Research and Practice
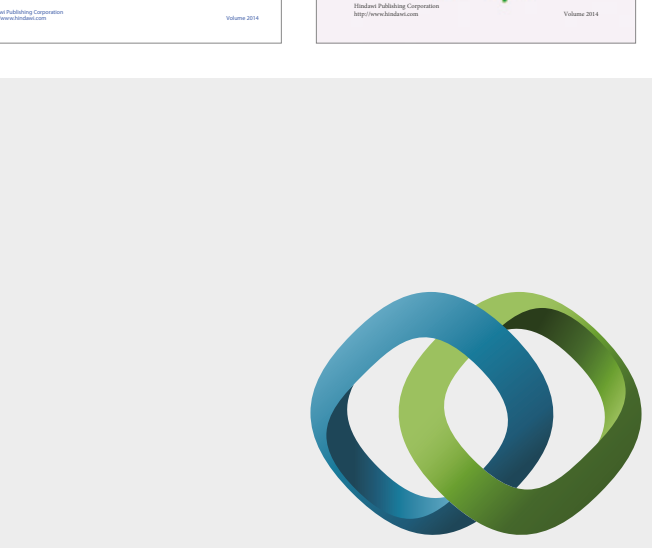

\section{Hindawi}

Submit your manuscripts at

https://www.hindawi.com
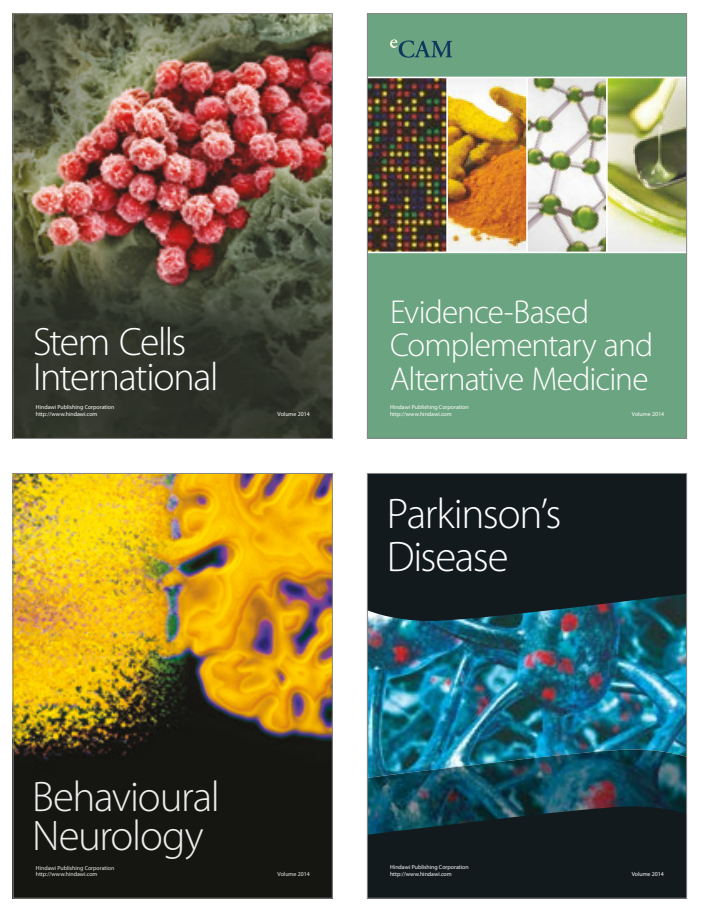
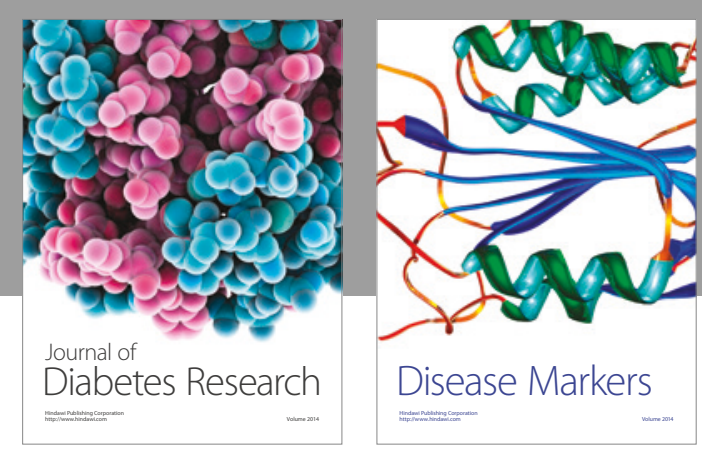

Disease Markers
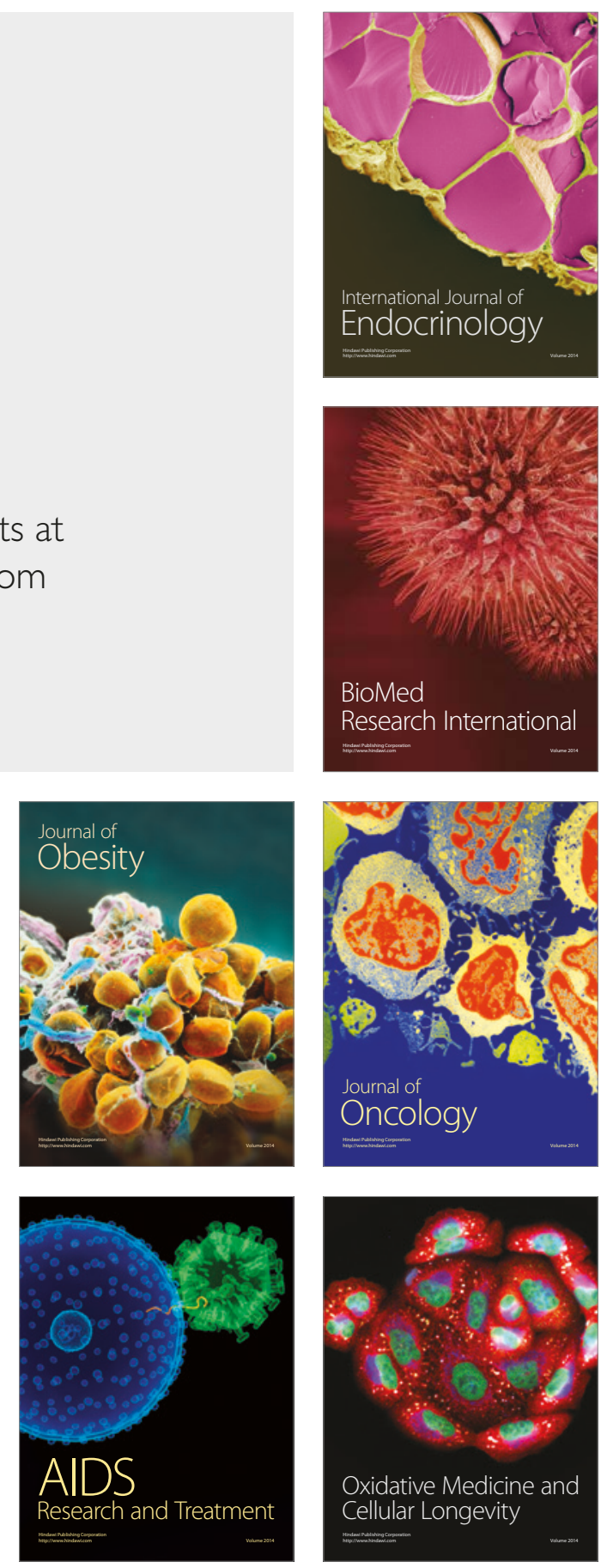